Instituto Internacional de Investigación y Desarrollo Tecnológico Educativo INDTEC, C.A.

DOI: https://doi.org/10.29394/scientific.issn.2542-2987.2017.0.0.16.295-316

OAI-PMH: http://www.indteca.com/ojs/index.php/Revista Scientific/oai

\title{
Aspectos de la Violencia Escolar que Afectan la Convivencia entre los Actores Socio-Educativos
}

\author{
Autores: Rosa Margarita Sáenz Rojo \\ Universidad Nacional Experimental "Rafael María Baralt”, UNERMB \\ rosa.saenz1905@gmail.com \\ Trujillo, Venezuela \\ Luz Marina Matheus Rojo \\ Universidad Nacional Experimental "Rafael María Baralt”, UNERMB \\ luzmarinamatheus24@gmail.com \\ Trujillo, Venezuela \\ Richard José Rodríguez Montilla \\ Universidad Nacional Experimental "Rafael María Baralt”, UNERMB \\ richardrodriguez777@gmail.com \\ Trujillo, Venezuela
}

\section{Resumen}

La investigación se realizó en la ETIR "Laudelino Mejía" Municipio Trujillo, Parroquia Cristóbal Mendoza Estado Trujillo. Venezuela. La no violencia, tanto en su teoría como en su práctica, supone para la UNESCO el rechazo de la violencia y busca la resolución de los conflictos y la realización de objetivos comunes desde una perspectiva constructivista. El objetivo de la investigación fue develar los aspectos de la violencia escolar que afectan la convivencia entre los actores socio-educativos. El estudio se orientó desde la etnografía, la cual se interesa en el proceso sistemático de aproximación a una situación social, considerada de manera global en su propio contexto natural. Información que se recolectó a través de entrevistas a profundidad y grupos focales. Posteriormente se transcribió y organizó la información para elaborar las diferentes categorías de análisis, Finalmente las conclusiones reflejan diferentes respuestas satisfactorias para una convivencia positiva. De igual manera supone promover instancias de colaboración entre docentes y estudiantes; comisiones, grupos de trabajo, con los que podamos ayudarles mejor y, a la vez, involucrarlos activamente en la promoción y difusión de una cultura de paz, que fomente el pluralismo cultural y el respeto a la diversidad.

Palabras clave: violencia; convivencia; cultura de paz. 


\title{
Aspects of School Violence Affecting Coexistence Among Socio- Educational Actors
}

\begin{abstract}
The research was conducted at the Industrial Technical School Robinsoniana "Laudelino Mejia" Municipio Trujillo, Trujillo State Parroquia Cristobal Mendoza. Venezuela. Nonviolence, both in theory and in practice, it is for UNESCO rejection of violence and seeks the resolution of conflicts and the realization of common objectives from a constructivist perspective. Throughout 2001, it said Agency launched the initiative of a collection of examples of "good practices" for conflict resolution in the school world, both formal and non-formal level. The purpose of the study was to reveal aspects of school violence affecting coexistence among socio-educational actors. Study was oriented from ethnography, which is interested in the systematic approach to a social situation, considered globally in their natural context. Information collected, transcribed and arranged to develop the different categories of analysis, so that school life as subjective category, subcategories emerged: coexist harmoniously rights responsibly, assertive communication. As relates to school violence emerged: indiscipline, damage to the institution, physical and psychological violence, teaching practice assertiveness. Ending with reflections.
\end{abstract}

Keywords: violence; coexistence; culture of peace. 


\section{Introducción}

Desde la década de los ochentas hasta hoy, en muchos países de Europa y América han sido notorios numerosos eventos violentos que ocurren dentro o en los alrededores de instituciones educativas, protagonizadas por estudiantes, donde inclusive se han presentado manifestaciones de violencia extrema; aunque en la mayoría de los casos las múltiples caras de las acciones de agresividad estudiantil no han alcanzado cifras extremas de muertes, éstas no se presentan excluidas, lo cual por su intensidad las ha convertido en tema de honda preocupación para los líderes educativos.

Las escuelas secundarias públicas de Latinoamérica han sido seriamente afectadas por el fenómeno del vandalismo, agresión grupal, disrupción en el aula, intimidación, entre otros eventos estudiantiles, resultando constante pérdida de bienes educativos, suspensión de clases que afecta el rendimiento estudiantil, daños físicos a actores escolares, y en fin, pérdida de la inversión del Estado.

La creación y difusión de la violencia en las instituciones educativas tienen causas múltiples y entrelazadas, inherentes tanto a la personalidad, familia, contexto social, los medios de comunicación, las relaciones interpersonales escolares, entre otros, por lo que hace necesario el estudio individualizado a cada una de estas.

Con frecuencia, la Organización de las Naciones Unidas para la Educación. La Ciencia y la Cultura (UNESCO, 2005) se refiere de manera explícita a la presencia creciente del fenómeno de la violencia escolar. Dentro de su sector de educación, ha desarrollado un programa de educación para la no violencia, que evoluciona constantemente. Para esta Organización internacional no consiste sólo en negar los sentimientos de cólera o de conflicto, sino que busca canalizar la energía subyacente hacia el objetivo de elaborar estrategias eficaces y respetuosas con los otros, excluyendo las actitudes de pasividad. 
Por su parte, El Fondo de las Naciones Unidas para la Infancia y la Adolescencia UNICEF, es reiteradamente sensible al fenómeno de la violencia en general contra los niños, las niñas y los adolescentes, y a su reflejo en el ámbito escolar. En el panorama sobre la violencia vinculada a la escuela, UNICEF ayuda a través de sus actuaciones y de sus propuestas a completar el análisis, llamando la atención sobre un problema previo, el de la violencia estructural, que impide a los niños y a las niñas acceder a la escuela.

En su Informe sobre la violencia y la salud (2003) se recoge la resolución de la Asamblea Mundial de la Salud de 1996, en la que se declaró que la violencia es un problema de salud pública fundamental y creciente en todo el recuadro. En dicha reunión se resaltaron las graves consecuencias de la violencia, y la necesidad de establecer actividades de salud pública para afrontar el problema.

A la hora de explicar y de comprender la naturaleza de la violencia, el Informe de la OMS plantea un enfoque o modelo ecológico que explora la relación entre los factores individuales y contextuales, y que considera la violencia como producto de muchos niveles de influencia sobre el comportamiento.

Por su parte, Venezuela presenta una preocupante situación similar a la planteada anteriormente, a menudo se suscitan hechos violentos dentro de diversos planteles educativos especialmente los de Educación Media General y Media Técnica Según información presentada por TELEVEN en el programa televisivo 100\% Venezuela transmitido en el mes de Junio del año 2004; en el 2001 se desarrollaron 182 manifestaciones estudiantiles con allanamientos en distintos planteles de Caracas asociados en algunos casos a la existencia de factores internos de la escuela concerniente con el clima organizacional, tales como relaciones interpersonales fracturadas, mala comunicación y conflictos.

El Centro Comunitario de Aprendizaje (CECODAP) durante el período Octubre 2008 - septiembre 2009, devela una serie de datos realmente 
alarmantes, producto del estudio realizado por la empresa Datanálisis. Para este organismo los datos más relevantes son los siguientes: a) 3.231 casos de NNA víctimas de algún tipo de violencia, del cual un $47,42 \%$ esté representado por la violencia social y un $26,25 \%$ por violencia escolar (en su estudio señalan que las escuelas ya no son un lugar seguro para los NNA, y este es sin duda uno de los datos más alarmantes). b) El 51\% por ciento de las víctimas de Violencia Social, corresponde al sexo masculino, siendo el homicidio la mayor de las causas halladas, en el cual el $90,43 \%$ de las víctimas eran adolescentes de entre 12 y 17 años.

Partiendo de lo referido anteriormente se plantean las siguientes interrogantes: ¿Cuáles son los rasgos más resaltantes que ocasionan los hechos de violencia escolar que afectan la convivencia escolar en la Escuela Técnica Robinsoniana "Laudelino Mejía" del estado Trujillo?, ¿Cómo afectan los hechos de violencia al entorno social donde está ubicada la Escuela Técnica Robinsoniana "Laudelino Mejía" del estado Trujillo? ¿Cuáles son los factores que inciden a participar en los hechos de violencia estudiantil en la Escuela Técnica Robinsoniana "Laudelino Mejía" del estado Trujillo?

Desde esta perspectiva, surge la presente investigación que utiliza el paradigma interpretativo con un enfoque metodológico etnográfico. Por ende, el objetivo del estudio fue develar los aspectos de la violencia escolar que afectan la convivencia entre los actores socio-educativos.

\section{Referentes teóricos}

La relevancia de esta investigación, se inicia sobre unos sólidos referentes teóricos que orientan y proporcionan importancia a la situación planteada 


\subsection{Teoría Humanista}

El humanismo surgió como una imagen del mundo al término de la Edad Media. Los pensadores humanistas fueron entonces intérpretes de nuevas aspiraciones humanas impulsados por la decadencia de la filosofía escolástica, cuyo centro de gravedad era la vida religiosa y la inmortalidad ultraterrena. El humanismo vino a sustituir esa visión del mundo con la reflexión filosófica abundante en productos racionales, en la que primaba la idea del hombre como ser humano, verdadero e integral.

De acuerdo con el Paradigma Humanista los alumnos son entes individuales, únicos, diferentes de los demás; personas con iniciativa, con necesidades personales de crecer, con potencialidad para desarrollar actividades y para solucionar problemas creativamente

Vale la pena tener presente a Carl Rogers. Como un estudioso entre quienes más han analizado el concepto de aprendizaje, Rogers afirma que el alumno promoverá su propio aprendizaje en cuanto éste llegue a ser significativo para él mismo. Esto sucede cuando en la experiencia se involucra a la persona como totalidad, cuando se incluyen sus procesos afectivos y cognitivos, y cuando, además, el aprendizaje tiene lugar en forma experimental.

En este sentido, reviste gran importancia que el alumno considere el tema a tratar como algo relevante para sus objetivos personales y que el aprendizaje se promueva con técnicas participativas, a través de las cuales el alumno tome decisiones, movilice sus propios recursos y se responsabilice de lo que va a aprender.

\subsection{Teoría Ecológica}

El modelo Ecológico es una estructura de múltiples niveles que incorpora tanto los factores personales como ambientales, que actúan sobre el ser humano. Trata de demostrar que no existe un nivel o causa única 
determinante de la conducta humana, la misma sólo puede explicarse dentro de un contexto y sus experiencias contextuales que afectan al desarrollo Para Bronfenbrener (1987), en una visión más específica dirigida al tema de las relaciones, toma la ecología como:

Una concepción teórica del ambiente como algo que va más allá de la conducta de los individuos y que incluye sistemas funcionales, tanto dentro como entre entornos, sistemas que también pueden modificarse y expandirse, contrasta ampliamente con los modelos de investigación vigentes (pág. 27)

Estos modelos establecidos se caracterizan por emplear una óptica científica que restringe, oscurece, e incluso ciega la visión que tiene el investigador de los obstáculos y las oportunidades del ambiente y del notable potencial de los seres humanos para responder de una manera constructiva a un medio compatible ecológicamente, cuando lo encuentran.

\subsection{Cultura de Paz}

La cultura de la paz definida por resolución de la ONU, en la Asamblea General el 6 de octubre de 1999 en el Quincuagésimo tercer periodo de sesiones, Acta 53/243, consiste en una serie de valores, actitudes y comportamientos que rechazan la violencia y previenen los conflictos tratando de atacar sus causas para solucionar los problemas mediante el diálogo y la negociación entre las personas, los grupos y las naciones, teniendo en cuenta un punto muy importante que son los derechos humanos, así mismo respetándolos y teniéndolos en cuenta en esos tratados.

En este documento titulado Declaración y Programa de Acción sobre una Cultura de Paz, la Asamblea General hace alusión y énfasis en la Carta de las Naciones Unidas, a la Constitución de la Organización de las Naciones Unidas para la Educación, la Ciencia y la Cultura, a la Declaración Universal 
de los Derechos Humanos y reconoce que "la paz no es solo la ausencia de conflictos".

\subsection{Convivencia}

La convivencia constituye la esencia de las relaciones sociales. El ser humano, por su naturaleza necesita de otros: es, se hace y construye en la relación con demás. En este orden de ideas, Maturana (1999) expresa algunas consideraciones sobre el papel de la educación para la convivencia al señalar que:

La tarea de la educación es crear un espacio de transformación en el convivir de las personas, para el vivir. No para servir una empresa, ni ninguna tarea en particular, porque el hacer un trabajo de una u otra clase, va a venir con el vivir (pág. 138)

En el mismo orden de ideas, en la convivencia es indispensable la independencia y la autoconfianza, pero en el discurrir de la vida no puede prescindirse del apoyo y de la compañía de los otros.

\subsection{Violencia Estudiantil}

Al respecto, Fernández (1999) la admite como "comportamiento de agresividad gratuita, injustificada y cruel, que denigra y daña tanto al agresor como a la víctima" (pág. 43). Considera que la misma hace uso deshonesto, prepotente y oportunista de poder sobre el contrario, sin estar legitimado para ello. En todo caso, la violencia es un acto que tiene como consecuencia evitar la realización de la satisfacción de las necesidades afectivas, materiales, y/o mentales de una persona por causa de otra. 


\section{Metodología}

\subsection{Paradigma cualitativo e interpretativo}

Desde esta visión, Martínez. (1999), afirma que la "concepción actual de paradigma no se refiere sólo a cada ciencia en particular, sino a la ciencia como modo de conocer, convirtiéndose en "principio rector del conocimiento y de la existencia humana" (pág. 23). Aquí juega un papel relevante la reflexión profunda sobre las experiencias vividas y las relaciones vitales que se comparten pues se constituyen en las preguntas que configuran la base para el conocer.

Al considerar la complejidad de las relaciones humanas en contextos sociales es imposible comprenderlos aplicando solamente metodologías que impliquen su fragmentación. Debido a las fuertes interrelaciones entre los miembros y a la multidireccionalidad de los comportamientos de los integrantes, es preciso acudir a un paradigma alternativo que ofrezca la posibilidad de abordar tanto las partes como el todo.

De acuerdo a lo planteado, el paradigma interpretativo de acuerdo con Pérez (2004), emerge como: “...alternativa al paradigma racionalista, puesto que en las disciplinas de ámbito social existen diferentes problemáticas, cuestiones y restricciones que no se pueden explicar ni comprender en toda su extensión desde la metodología cuantitativa" (pág. 26). En este sentido, su objeto es el desarrollo de conceptos que ayuden a comprender los fenómenos sociales en medios naturales dando la importancia necesaria a las intenciones, experiencias y opiniones de todos los participantes.

\subsection{Método de Investigación: La Etnografía}

A los fines de la presente investigación se seleccionó el método etnográfico. Éste, de acuerdo con Martínez (2008), consiste en la producción de estudios analítico-descriptivos de las costumbres, creencias, prácticas 
sociales y religiosas, conocimientos y comportamientos de una cultura particular, razas o pueblos.

La intención básica de toda investigación etnográfica es naturalista, es decir, trata de comprender las realidades actuales, entidades sociales y percepciones humanas, así como existen y se presentan en sí mismas, sin intrusión alguna o contaminación de medidas formales o problemas preconcebidos

\subsection{El trabajo de campo y la recolección de los datos}

El trabajo de campo se da inicio con las tareas previstas desde el primer día en el que el investigador se encuentra en el espacio de estudio. En este caso, el periodo del trabajo de campo se extendió durante el año escolar 20142015. La permanencia en la institución fue un proceso paulatino de acercamiento e interiorización, principalmente para conocer la cotidianidad escolar, sus actores y las actividades habituales de los escolares, entre otros. Permitiendo las interacciones con algunos escolares quienes, a su vez, se convirtieron en informantes. Ellos contextualizaban los hechos y sucesos de violencia / agresión / maltrato entre pares; en segunda instancia, ayudaron a identificar a los agresores y a las víctimas. Asimismo, en ese proceso, se construyó una relación próxima con los obreros que reconstruyeron situaciones y casos de indisciplina, vandalismo y violencia ocurridos en la escuela. En algunos casos, esas historias fueron narradas como anécdotas.

Iniciado el trabajo de campo en la escuela se visibilizan los hechos de violencia a tal punto que suspenden las clases por tres semanas. Esto da fundamento a ir definiendo el concepto de 'acoso escolar' o violencia entre pares, que abarca formas de intimidar, atemorizar, excluir, fastidiar, incomodar, provocar, desafiar, golpear o insultar. Esta definición ha orientado el camino que se siguió para develar la violencia escolar a través de las técnicas e instrumentos cualitativos. Se comenzó con algunas entrevistas 
semi-estructuradas dirigidas al personal de la escuela (directores, profesores, Coordinador pedagógico y de evaluación, obreros), entrevistas a grupos focales las cuales se realizaron por año y sección. La entrevista permite complementar y verificar la información obtenida mediante la observación participante. El etnógrafo no necesariamente tiene que entrevistar a todas las personas relacionadas con su estudio; para ello recurre a algunos informantes claves. La entrevista puede ser casual e informal o estructurada. Los datos recabados a través de la entrevista pueden ser registrados en forma de notas durante o una vez culminada la entrevista, o también es posible utilizar la grabación en audio o video de una forma abierta.

Las conversaciones casuales con los estudiantes han permitido abordar el tema desde narraciones de sus propias historias. Por otro parte se determinó dos tipos de limitaciones: en primer lugar, la dificultad de entender básicamente el problema del bullying por el personal de la escuela donde la violencia resalta siempre como algo exógeno a la cultura escolar, desde la perspectiva institucional. En ese sentido, los educadores y autoridades han realizado fuertes críticos a la funcionalidad de la escuela como un ámbito fundamental de educación. En segundo lugar, el poco tiempo disponible de los profesores para ser entrevistados: tienen que ir a otros centros de estudios a laborar.

En ese escenario, se optó por el uso de la observación participante, particularmente en torno a las acciones cotidianas de los escolares: dentro de la escuela, se genera un sinfín de acciones, comportamientos y comunicaciones que deben ser sistematizados como información para luego estructurarse en datos. Para ello, se elaboró una guía de observación a través de la cual se exploró una serie de indicadores (características de las relaciones interpersonales, finalidad del ejercicio de la violencia, actores de la violencia, momentos y espacios de la violencia, contenidos de discurso, entre otros) y se 
efectuó un mapeo de los espacios de violencia al interior de la escuela, es decir un seguimiento visual de las situaciones de acoso y violencia escolar.

Por ende, los registros etnográficos se realizaron por espacios cortos; a su vez, fueron permanentes, estableciéndose secuencias y frecuencias particularmente en el transcurso del medio día, tiempo en el cual ocurrían con mayor frecuencia los hechos violentos. En ese escenario, la observación no participante se realizó en tres momentos diferentes: el ingreso al establecimiento escolar, la salida y en la avenida. De esta manera, se pudo llevar a cabo un trabajo de campo con una presencia sistemática y una prolongada permanencia con el grupo social estudiado, con un modo de observación más preciso y restringido, dedicado al registro de información y datos.

El interior de la institución y la avenida han sido un terreno fundamental para desarrollar la observación; momentos en los que el observador accedió a las estructuras del significado de la violencia mediante descripciones densas de los hechos. En la escuela se desencadenan conflictos sin aparente motivación o finalidad, se da la presencia de encapuchados; las estructuras formales de intervención poco o nada pueden hacer, pues las formas de transgresión no solo avasallan las posibilidades del control de los profesores por su cantidad y recurrencia, de igual manera se ven amenazados su integridad física; además, se dan en los espacios como en la avenida donde se observa los enfrentamientos con las fuerzas policiales, obstaculizan el tráfico automotor, amenazan a los transeúntes, quema de cuchos entre otros.

La observación es un modo original de conocimiento que permite descubrir las particulares de una sociedad o cultura. Para la sociología, la observación es parte inherente a las metodologías propias de la investigación cualitativa. Es cierto que su uso ha sido ampliamente difundido por la antropología, pero la sociología la emplea también para realizar estudios específicos. En antropología, se recurre a la observación para ver diferencias 
entre culturas antiguas y modernas o entre diferentes pueblos y principalmente sus pautas culturales; para la sociología, en cambio, contribuye a estudiar las pautas de interacción entre miembros de un grupo (los símbolos y signos que construyen grupalmente) y que les dan un sentido de pertenencia (conductas, gestos, lenguajes, entre otras).

Otros de los pasos requeridos en la investigación es la triangulación, la cual permite reinterpretar la situación en estudio, a la luz de las evidencias provenientes de todas las fuentes empleadas en la investigación. Constituye una técnica de validación que consiste en "cruzar", cualitativamente hablando, la información recabada.

En sociología, las técnicas e instrumentos de recolección tradicionales son las encuestas, entrevistas y las historias de vida, por lo tanto, son más aceptadas y son reconocidas como válidas y objetivas. En cambio, la observación no participante o etnografía directa es una opción de reciente uso en el ámbito de estudio sociológico. No obstante, muchas temáticas no solo pueden ser investigadas o aprehendidas desde las técnicas tradicionales. Por ejemplo, la enseñanza-aprendizaje en la escuela, el manejo y ocupación del espacio por estudiantes/jóvenes, la construcción de la masculinidad de los adolescentes son tópicos eminentemente cualitativos, por tanto, deben ser estudiados desde la etnografía ya que implican una diversidad de ritualidades en su desenlace que pueden ser captadas mediante la observación.

\section{Análisis e Interpretación de la Información}

Convivir y coexistir en armonía con sus semejantes y en equilibrio con la ecología, ha sido; desde los albores de la historia terrenal, salvo algunas excepciones espaciales y temporales, una dificultad para la especie humana. El hombre conquistó el espacio exterior, fue capaz de llegar a la luna, descubrió nuevos planetas y encontró la curación milagrosa para terribles enfermedades. De igual manera, gracias a sus habilidades físicas y mentales, 
revolucionó la ciencia y la tecnología inventando maravillosas obras como el automóvil, los aviones, las telecomunicaciones, la informática, la electrónica, entre tantas obras tangibles y necesarias.

Sin embargo, pese a sus dotes de inteligencia y conocimientos científicos, el hombre no ha hallado la paz plena y duradera. Durante siglos la humanidad ha experimentado la violencia como parte insoslayable de su existencia. La historia del hombre registra numerosos hechos de violencia, evidenciando su presencia en cada sociedad, por pequeña o grande que sea, generando cuantiosas pérdidas humanas, ecológicas y materiales.

El hombre, en su afán de poder, riqueza y producción material dejó a un lado dos necesidades básicas, valores esenciales para la subsistencia, como son el amor y la paz, principios rectores que se traducen en tolerancia, comprensión, respeto mutuo, solidaridad y el bien común.

En este sentido Boggino (2004) afirma que, para disminuir la violencia, por no decir erradicar, se requiere visualizar lo encubierto, una reconsideración y una transformación de la concepción sobre la violencia, y por consiguiente, de los mecanismos de intervención.

Desde esta perspectiva, las observaciones registradas por el investigador se centran primordialmente en los comportamientos físicos y verbales de los estudiantes en sus respectivos escenarios:

Lo observado desde la Avenida: Es frecuente que, en horas de mediodía, el paso por la Avenida Laudelino Mejías, entrada principal a Trujillo este obstaculizado debido a que en las inmediaciones de la institución se encuentran personas encapuchadas quemando cauchos, lanzando piedras, colocando cualquier tipo de objetos en la vía. La policía, particularmente los motorizados, se presentan en los puntos de la avenida, que marca las intersecciones de éstas con rutas alternas, con la finalidad de desviar el tráfico hacia esas vías para asegurar el libre tránsito. Estas manifestaciones son 
cortas, y en ciertas ocasiones son como intermitentes, pues los manifestantes desaparecen y el tráfico se reanuda y, luego, aparecen nuevamente los manifestantes y el tráfico vuelve a interrumpirse. Esto ocurre generalmente entre las doce del mediodía y las dos de la tarde.

A lo largo de la avenida, los estudiantes se movilizan en grandes grupos, se conforman grupos de ambos sexos, en los que predomina el color azul de la camisa. Se oye el grito, la algarabía y el lanzamiento de piedras que tiene como blanco el vidrio de algún vehículo. Además, se ha convertido en una conducta común detener por parte de los estudiantes a las camionetas con batea y obligar o persuadir al conductor a transportarlos.

Lo observado desde el interior de la Institución: En el patio principal se observan presuntos estudiantes con el rostro cubierto con franelas, que se desplazan a la entrada principal y otros lanzan piedras a través del pasillo principal, en ese momento el personal docente se dirige hacia las oficinas y cierran las puertas, los estudiantes corren en grandes grupos hacia la entrada principal para salir de la institución. Se inicia el sonido de bombas explosivas $y$ en ocasiones gas lacrimógeno; algunos docentes observan desde las ventanas de las oficinas, mientras que otros llevan sus vehículos a ocultarlos dentro de las aulas talleres.

La opinión de algunos estudiantes: De acuerdo a algunos estudiantes entrevistados luego de una Asamblea, opinaron que: "La huelga la inicia los cuarto, quinto y sexto año y dicen que protestan por el comedor, los baños sucios y precios de la cantina o simplemente porque solo les provoca salir a la calle". Mientras que los alumnos del segundo y tercer año, manifestaron: "No sabemos el porqué de las huelgas"; ellos participan porque "siguen a la mayoría".

Habla el Representante de la Zona Educativa: "El año pasado dijeron que era el Director, se cambió; que era dotación, se dotó; que eran infiltrados y que era a las horas del mediodía, se dotó de equipos deportivos y sigue la 
misma situación. Entonces se formulan una serie de interrogantes que den respuesta a las causas de los disturbios y hechos violentos.

Hablan los profesores: Se tienen identificados a los revoltosos de las once de la mañana y el grupo interno los conoce y apoya.

No es culpa de los profesores, aunque digan aquí y en las busetas que no queremos dar clases. El año pasado se aprobó el año escolar con un 35\% del contenido de las asignaturas: El Consejo Docente para este año decidió y eso consta en acta, que no van a aprobar con el $35 \%$, pero tampoco vamos a trabajar en agosto ni sábados y domingos para los 180 días. El problema es a la hora del almuerzo, primero come Automotriz y Mantenimiento, mientras que a Construcción Civil no lo dejan comer.

Habla el Jefe del Municipio: Hay que invitar para la próxima reunión a los voceros estudiantiles y propone: Que se cambie el horario de 7:00 am 11:40 am, más una rotación de horario de almuerzo cada 15 minutos: Programar juegos intercursos, reactivar el campo deportivo trayendo a un nuevo profesor. Reprogramar el año escolar y ubicar unidades de seguridad pública (Guardia Nacional y Policía uniformada) en la parte externa de la institución: En el interior de ésta, los padres y representantes "contribuyendo con la paz estudiantil" actuando como vigilantes.

En el marco de la investigación desarrollada, los docentes y los estudiantes le atribuyen varias interpretaciones de los cuales se parte. Ante los hechos de violencia e indisciplina de los estudiantes, los docentes y la institución argumentan que el estudiante siempre es el responsable (la ritualización de la transferencia de culpabilidad). Uno de los argumentos más frecuentes en la institución teniendo como eje los docentes, es que se ha llamado: "la situación del adolescente-rey". Los docentes manifiestan que: "La LOPNNA los protege de "todo" y a pesar de lo que hagan no sirve de nada sancionarlos". "Antes uno expulsaba al que se portaba mal, ahora no podemos", "los alumnos nos amenazan con la LOPNNA". 


\section{A modo de Conclusión}

La violencia entre pares en la escuela no ha sido un fenómeno sencillo de abordar, independientemente de la metodología empleada. En ese sentido, se destacar, por un lado, la flexibilidad del investigador y por otro, la aplicación de la técnica de observación participante como metodología de abordaje de la investigación.

La flexibilidad es una condición que debe poseer el investigador social pese a que las investigaciones no todas son iguales. En ese proceso, se presentan dificultades (teóricas, prácticas y metodológicas) que deben ser resueltas de manera oportuna durante la investigación y/o trabajo de campo. Barragán (2003) dice que el investigador es el instrumento directo a través del cual se obtiene la información y no así los cuestionarios.

Desde el punto de vista social, esta investigación ayuda a describir los brotes de agresividad o de violencia en los centros educativos, que constituyen un fenómeno cada vez más frecuente, el cual se interpreta como un reflejo de lo que ocurre en la sociedad. Se señala que las acciones antisociales exhibidas por los adolescentes muestran lo que ocurre en su entorno, y sus causas hay que buscarlas en varios factores: sociales/ ambientales, relacionales, escolares, familiares y personales.

La familia como primer ámbito educativo necesita reflexionar sobre sus pautas educativas y tomar conciencia de su papel en la educación de sus hijos e hijas. La realidad actual se le escapa, y esto repercute en la vida del niño y la niña, lo cual conlleva a su vez problemas escolares y familiares que surgen a diario: desinterés, falta de motivación, dependencia, bajo rendimiento, fracaso escolar, violencia, etc., y no se pueden achacar a la sociedad en abstracto, a la familia, a la escuela o al alumnado, de manera independiente, sino que la interacción de todos ellos es la que propicia esta situación.

Son los padres y las madres quienes gozan de una relación de intimidad única que exclusivamente se da en el seno de la familia y que permite todo 
tipo de interrelaciones personales: de afecto, ayuda, orientación, soporte, entre otros, que influyen y modifican los comportamientos de todos sus miembros. Suele decirse que en una familia todos educan y son educados.

Ahora bien, en relación a la violencia, no es suficiente considerarla simplemente como un comportamiento social indeseable. Con el fin de abordarla con más o menos probabilidad de éxito es necesaria una comprensión más amplia. Frente a la amenaza que representan actualmente los actos violentos, la puesta en práctica de medidas coercitivas y, a veces, punitivas, inmediatas, es por supuesto, comprensible y razonable en una escuela que fue tomada por sorpresa y para lo cual no está preparada.

De esta manera, buscar comprender los actos de violencia, analizando la vida de los culpables, sin duda explica en cierta manera el problema, pero no consigue explicar y resolver completamente el enigma del acto. Es necesario resaltar que los actos violentos cometidos por los adolescentes de esta institución, es que, además de la falta de claridad de sus móviles, estos no tienen en cuenta las consecuencias de sus actos contra las víctimas. Sean estas, profesores, transeúntes o la institución misma. Esto da cuenta de que la escuela está débil en cuanto a la formación de ciudadanía.

Desde la perspectiva metodológica de la investigación cualitativa se asume la realidad como un fenómeno interpretativo de los significados que se construyen en la vida cotidiana, ya que el valor de la investigación involucra perspectivas epistemológicas sobre la objetividad y la subjetividad (Barragán, 2003). En ese sentido, la técnica de la observación implica reconocer posiciones epistemológicas sobre la posición del método de investigación desde paradigmas y enfoques (Valles, 2007).

\section{Referencia}

Barragán, R. (coord.) (2003). Guía para la formulación y ejecución de proyectos de investigación. La Paz: PIEB. 
Boggino, N. (2004). Los Valores y las Normas Sociales en la Escuela. Rosario Santa Fe. Argentina: Homo Sapiens Ediciones.

Brofenbrenner, U. (1987). La Ecología del Desarrollo Humano. Barcelona: Paidos.

Centro Comunitario de Aprendizaje. CECODAP (2008-2009). Estudios sobre

la violencia contra niños, niñas y adolescentes en Venezuela. Informe al experto independiente de la Secretaria de las Naciones Unidas Venezuela. Caracas.

Fernández, I. (1999). Prevención de la violencia y Resolución de conflictos. Madrid: Narcea, S.A. de Ediciones.

Martínez M. (2008). La investigación cualitativa etnográfica en educación. Manual Teórico Práctico. México: Trillas.

Maturana, H. (1994). Formación Humana y Capacitación. (2da. Ed). Santiago: Editorial Dolmo.

Pérez, E. (2005). Educar para Humanizar. Editora Estudios CA. Venezuela. ONU. (1999). Educación para la Paz. Madrid, Síntesis.

Organización de las Naciones Unidas para la Educación. La Ciencia y la Cultura UNESCO (2001). Violencia escolar: Perspectiva de las Naciones Unidas. Revista Ibero-Americana de Educación, N. 38. Recuperado de: http://www.rieoei.org/rie38a01.htm

Organización Mundial de la Salud (2003). Informe mundial sobre la violencia y la salud. Ginebra, OMS.

TELEVEN (2004). Programa televisivo 100\% Venezuela. transmitido en el mes de junio. Canal TELEVEN. Venezuela.

Valles, M. (2007). Técnicas cualitativas de investigación social. Madrid: Síntesis. 


\section{Rosa Margarita Sáenz Rojo \\ e-mail: rosa.saenz1905@gmail.com}

Doctora En Educación. UNERMB Trujillo. Magister

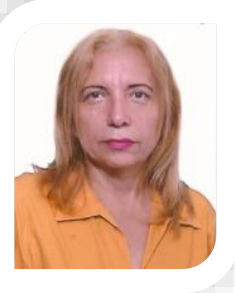
Administración de la Educación Básica. UNERMB Estado Trujillo. Licenciada en Educación Integral (Área de concentración Ciencias Sociales). Universidad Nacional Experimental "Simón Rodríguez". Núcleo Valera, Trujillo. Diplomado en Cultura de Paz Cátedra de la Paz ULA. Coordinadora Regional de Defensoría Educativa. Coordinadora Regional de Educación Intercultural desde enero 2012 hasta la presente. Profesora invitada de la Universidad Alonso de Ojeda. Tutora y evaluadora de Trabajos de Investigación en posgrado. Ponente de la investigación y Visibilización de las Comunidades Indígenas En el estado Trujillo. Pedagogía 2015, la Habana 30 de enero de 2015. 
Licenciada en Educación Integral Mención Ciencias

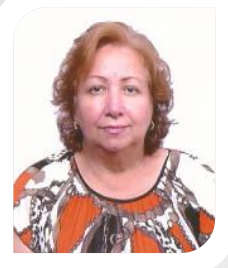

Sociales (Universidad Nacional Abierta), Magíster en Administración de la Educación Básica (UNERMB), Doctorado en Educación (UNERMB), Diplomado en Administración Cultural en Venezuela (Asociación Venezolana de Gerencia y Desarrollo Cultural. CONAC), Especialización en Desarrollo de las Habilidades y Actitudes Gerenciales. (Gerencia y Gestión Local. PNUD. Gobernación Trujillo), Coordinadora de Formación en investigación (PRIGED), Maestra tutora Misión Sucre de la Aldea Universitaria Ciudad de Valera (UBV), Maestra tutora de Prácticas Educativas (UNEFA), Profesora Invitada en el Eje de Investigación de la Maestría Administración de la Educación Básica (UNERMB Trujillo), Tutora de trabajos de investigación en Posgrado. 


\section{Richard José Rodríguez Montilla}

e-mail: richardrodriguez777@gmail.com

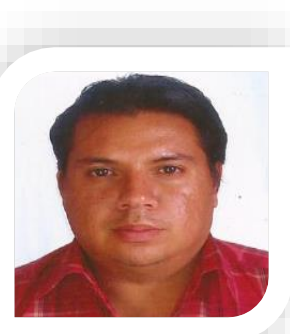

Profesor en la especialidad de educación integral (Universidad Pedagógica Experimental Libertador, extensión Trujillo). Magíster Scientiarum en Administración de la Educación Básica (UNERMB, extensión Trujillo). Doctor en Educación (UNERMB, extensión Trujillo). Abogado (Universidad Bolivariana de Venezuela, Pampanito). Diplomado en Ciencias Jurídicas (Colegio de Abogados del Estado Trujillo). Expresidente de la Comisión de Educación del Consejo Municipal de Pampanito (CMP). Docente de aula y coordinador de protección y desarrollo estudiantil (E.B. Gral. Manuel Gogorza Lechuga, Pampanito). Ponente, vocero y orientador en el congreso pedagógico (municipal, intermunicipal y estadal, Trujillo).

El contenido de este manuscrito se difunde bajo una Licencia de Creative Commons ReconocimientoNoComercial-Compartirlgual 4.0 Internacional 